\title{
Research on the dynamometer temperature characteristics of magneto-rheological fluid dynamometer
}

\author{
Fang Yi*, Luo Yiping, Ren Hongjuan \\ College of Automotive, Shanghai University of Engineering Science, Shanghai, 201620, P.R. China \\ Email address: \\ 812155164@qq.com (Fang Yi), lyp777@sina.com (Luo Yiping), ren-hongjuan@163.com (Ren Hongjuan)
}

\section{To cite this article:}

Fang Yi, Luo Yiping, Ren Hongjuan. Research on the Dynamometer Temperature Characteristics of Magneto-Rheological Fluid Dynamometer. Science Research. Vol. 2, No. 6, 2014, pp. 185-189. doi: 10.11648/j.sr.20140206.16

\begin{abstract}
The dynamometer measuring device through the magnetic rheological fluid self-designed, analyzes the basic characteristics of magneto rheological fluid, and then through the experimental study on the temperature characteristics of magneto rheological fluid. Dynamometer with magneto rheological fluid (MRF) was tested, in different conditions, when the system reached the steady state, temperature changes in the key points of the rotary. And through the analysis, the temperature distribution of the device was obtained, which provides the theoretical basis for the effect of temperature on transmission performance. Then, the obtained results, which can provided a good reference for the design of MRF gearing to optimum design and intelligent cooling system.
\end{abstract}

Keywords: Magneto Rheological Fluid, Dynamometer, Temperature Characteristics

\section{Introduction}

Magneto-rheological fluid ${ }^{[1]}$ is a new material with the future development and application value of Engineering (smart materials), good performance of magneto rheological fluid under the action of the magnetic field can generate magnetic rheological effect obvious, namely fast reversible transformation in the liquid and solid, the conversion is completed in a millisecond. In the process, the viscosity of magneto rheological fluid was continuously, also it was changed steplessly, The conversion process is very fast, and controllable, minimal energy consumption, also can realize the real-time control ${ }^{[2]}$.

Research on MRF was developed on the basis of electro-rheological fluid $(\mathrm{ERF})^{[3]}$ together. Generally, Winslow $^{[4]}$ found ER phenomenon in twentieth Century at the end of 40's, and this phenomenon explained by chain structure formed electric field induced solid particle interaction hindered shear flow. He also mentioned ER of magneto rheological fluid which is similar with phenomenon MRF.

It is commonly accepted that the study of the earliest of magneto rheological fluid begins with Rabinow ${ }^{[5]}$. Magneto-rheological fluid shear yield stress larger an order magnitude than the electro-rheological liquid, and magneto rheological fluid with good dynamic and temperature stability. Hence, the MRF has caused extensive concern in recent years.
With the improvement of the properties of magneto-rheological fluid and the development of the research, magneto rheological technology began to be used in the aerospace and mechanical engineering, automotive engineering, precision machining engineering, control engineering and other fields. It can be predicted that intelligent materials this good will lead to dramatic change of industrial technology in the next few decades. Magneto-rheological fluid and the magneto-rheological device has a great market prospect.

\section{The Structure of Magnetic Rheological Fluid and Its Temperature Characteristics}

Magneto rheological fluid is generally consists of three parts: ferromagnetic easy magnetization of solid particles ${ }^{[6]}$, liquid carrying oil ${ }^{[7]}$ and stabilizer ${ }^{[8]}$. Magnetic solid particles are made of spherical metal and ferrite magnetic materials generally (The particle size range of 5 50um). And as the carrier liquid oil continuous carrier, it is usually a good non-magnetic oil, such as mineral oil、Silicone oil、Synthetic oil etc. Good loaded liquid with zero field low viscosity, good stability at high temperature, no environmental pollution and other characteristics. The stabilizer is used to ensure that the 
particles are in liquid. Stability associated with magneto rheological fluid has a reunion stability and sedimentation stability, the former to prevent particles are bonded together, and the latter to ensure that the particle with time not to precipitate, stabilizer must have special molecular structure, is provided with a magnetic particle interface to produce highly dear and force pinning function group, the other end of the elastic groups also the need for a very easily dispersed in a base fluid to the appropriate length ${ }^{[9]}$.Schematic diagram of magneto rheological fluid loading device:

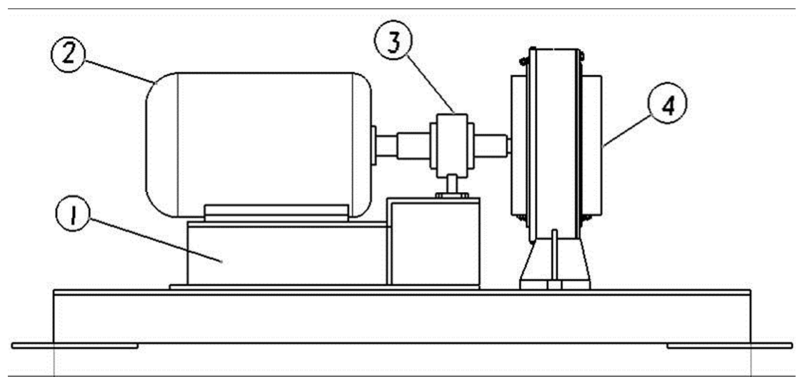

Fig. 1. Magneto-rheological fluid dynamometer bench diagram.

1. frame; 2. rotating member ;3. torque sensor; 4. MRF dynamometer device

\section{The Working Principle of Magneto Rheological Fluid Dynamometer}

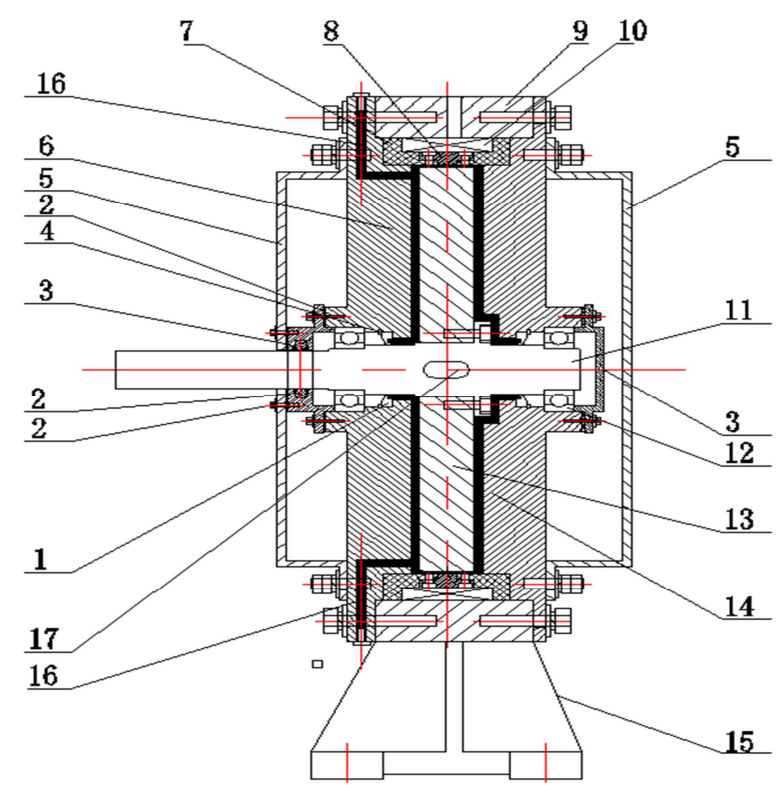

Fig. 2. Magneto rheological fluid dynamometer structure diagram.

1: skeleton oil seal; 2 : floating ring seal; 3 : bearing end cover; 4 : hole card reed; 5:cooling water jacket; 6: left shell; 7:the coil bracket; 8: isolation ring; 9 : the outer ring; 10: coil; 11: the rotating shaft; 12 : bearing; 13: the turntable; 14 : the right shell; 15: a base; 16: magneto-rheological fluid injection (discharge) ;17: a type key hole;

The formula for calculating power of the rotating parts:

$$
\mathrm{P}=\frac{\mathrm{nT}}{9550}
$$

Where, P:Power (KPa); n: speed(r.min-1); T: torque $(\mathrm{N} \cdot \mathrm{m})$;

As to formula 1, as long as they get the speed and torque of the rotating parts, the power size of the rotating parts can be obtained.

At present, the magneto rheological fluid constitutive properties of the most common view is that the magneto rheological fluid approximation considered as Bingham fluid model. Bingham model can successfully describe the mechanics model of steady shear conditions:

$$
\begin{array}{ll}
\tau=\tau_{\mathrm{y}}(\mathrm{B}) \operatorname{sgn}(\dot{\gamma})+\eta \dot{\gamma} & |\tau| \geq \tau_{\mathrm{y}} \\
\dot{\gamma}=0 & |\tau| \leq \tau_{\mathrm{y}}
\end{array}
$$

Where, $\eta$ is the viscosity coefficient of magneto-rheological fluid; $\dot{\gamma}$ is the shear strain rate; $\tau_{\mathrm{y}}(\mathrm{B})$ is dynamic yield stress, and with the increase of the magnetic induction intensity Bo and increased.

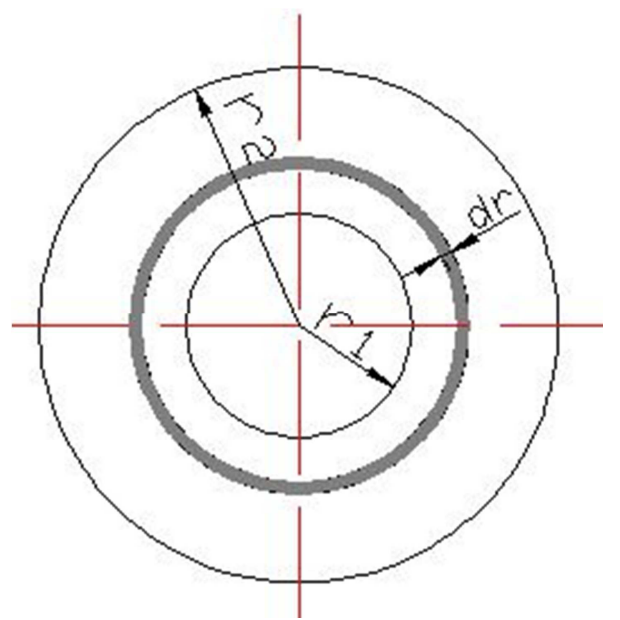

Fig. 3. The calculation model of the rotary magneto-rheological transmission.

In the figure, the working gap between the two rotary filled with magneto rheological fluid, its inner and outer diameter were R1 and R2, magneto rheological fluid thickness is h, small and large disk speed respectively $\omega 1$ and $\omega 2$, take the micro section $d r$ in work clearance at radius $r$, the volume of $\mathrm{dv}$ and the transmission of torque $\mathrm{dM}$ respectively

$$
\begin{aligned}
& d V=2 \pi r h d r \\
& d M=2 \pi r^{2} \tau d r
\end{aligned}
$$

To integral for formula (3), and ignore the magneto-rheological fluid distribution in each working gap inhomogeneity and the magnetic rheological fluid transmission efficiency, and the $\mathrm{n}$ slip power working space can be expressed as

$$
\mathrm{P}=\frac{2 \mathrm{n}}{3} \pi \tau\left(\mathrm{r}_{2}^{3}-\mathrm{r}_{1}^{3}\right)\left(\omega_{2}-\omega_{1}\right)
$$

The heat source of magneto-rheological transmission device mainly comes from the power loss of the friction heat, 
and coil between magnetic particles, the friction heat of the bearing and the rotating seal ring. Typically, the bearing and the rotating seal ring, the heating capacity is very small, and the coil is far from the working gap, both are less impact on the distribution of magnetic rheological fluid temperature which can be ignored in the analysis.

According to the basic theory of heat transfer, transmission loss of slip power all for the rise of internal temperature, andin the heat of the working gap radius $r$ at the rate of:

$$
\mathrm{Q}=\frac{\mathrm{dM}\left(\omega_{2}-\omega_{1}\right)}{\mathrm{dV}}=\frac{3 \mathrm{Pr}}{2 \mathrm{n} \pi \mathrm{h}\left(\mathrm{r}_{2}^{3}-\mathrm{r}_{1}^{3}\right)}
$$

Magneto-rheological fluid temperature field is a function of coordinates and time, which belongs to the nonlinear transient problem, then the cylindrical coordinates of magneto rheological fluid transient heat conduction equation ${ }^{[10]}$ is:

$$
\rho_{\mathrm{m}} \mathrm{c}_{\mathrm{m}} \frac{\partial \mathrm{T}_{\mathrm{m}}}{\partial \mathrm{t}}=\lambda_{\mathrm{m}}\left(\frac{\partial^{2} \mathrm{~T}_{\mathrm{m}}}{\partial \mathrm{r}^{2}}+\frac{1}{\mathrm{r}} \frac{\partial \mathrm{T}_{\mathrm{m}}}{\partial \mathrm{r}}+\frac{1}{\mathrm{r}^{2}} \frac{\partial^{2} \mathrm{~T}_{\mathrm{m}}}{\partial \varphi^{2}}+\frac{\partial^{2} \mathrm{~T}_{\mathrm{m}}}{\partial \mathrm{z}^{2}}\right)+\mathrm{Q}
$$

$\rho_{\mathrm{m}}$ The density of the material of magneto rheological fluid,

$\mathrm{c}_{\mathrm{m}} \longrightarrow$ Magneto-rheological fluid specific heat capacity

$\lambda_{m}$---Magneto rheological fluid thermal conductivity,

$\mathrm{T}_{\mathrm{m}}$---Magneto-rheological fluid temperature

$t$---Sliding time,

$z$---The axial size of magnetic particle layer where,

$\varphi$---Magneto-rheological fluid rotating plane and polar axis center angle

\section{The Experimental Scheme of Temperature Characteristics}

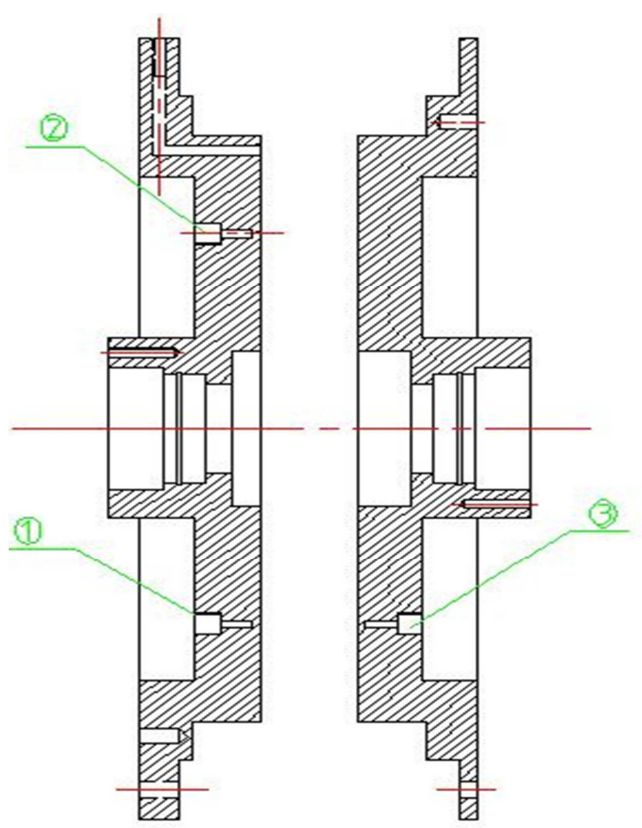

Fig. 4. Power measuring casing at the sensor arrangement.
Three points are selected for temperature sensor In this figure, (1) is the front temperature 1, (2) is for the front end temperature 2, (3) is the back-end temperature, (4) is for outlet temperature(it did not draw on the map, which is at the exit of measuring drainage dynamometer system)

In order to clearly understand the fever degree of each part of loading device, thus with better able to install water cooling system, so we conducted a series of experiments and studied the transmission under different conditions of fever.

The following charts are averaged after many experiments and results.

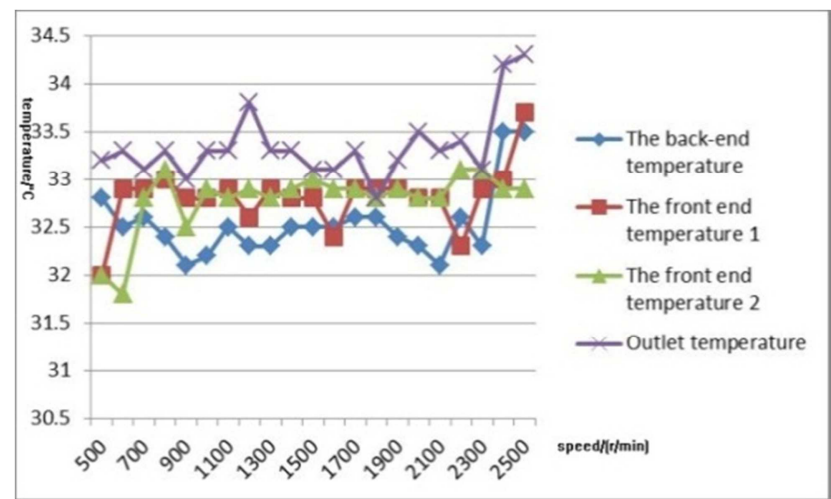

Fig. 5. Without magnetic rheological fluid.

At the condition without magneto-rheological fluid, by increasing the speed, the increase of the experimental point temperature is not obvious.

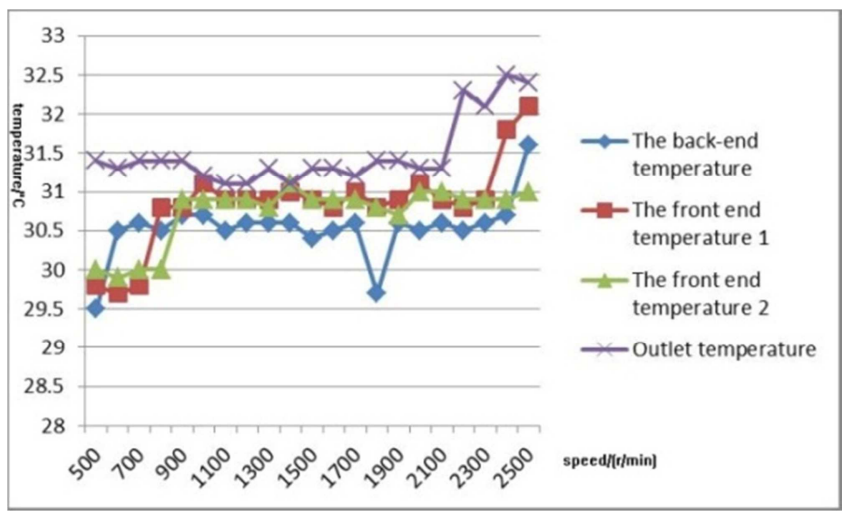

Fig. 6. With magnetic rheological fluid.

With magneto rheological fluid, this is not available in the current conditions, the temperature change is not very big, the two temperature measuring points (1) and (4) are more higher than the rest of temperature points.

With magneto rheological fluid, constant current (0.15A) conditions, increase the speed, the temperature change of the four measured points is obvious, especially points (1)、(2) and (3). The magnitude of temperature increase is relatively large 


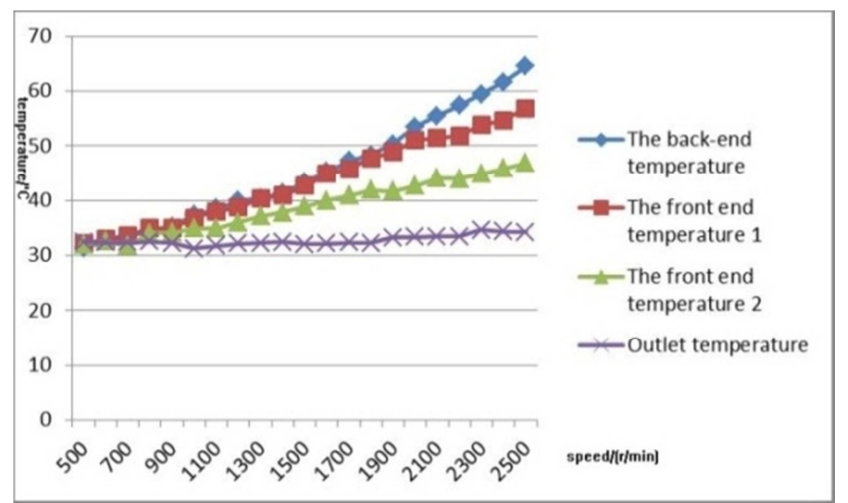

Fig. 7. Fixed current and increase speed.

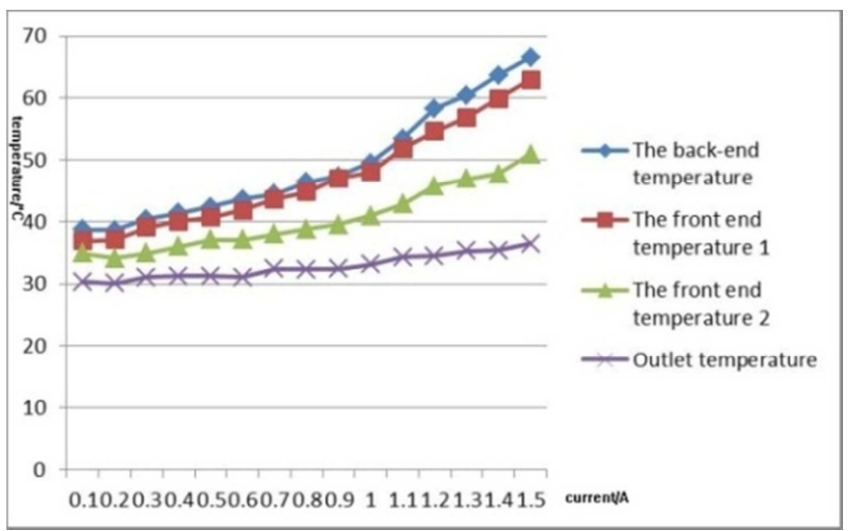

Fig. 8. Fixed speed and increase current.

With magneto rheological fluid, constant speed (1800r/min) under the condition of increasing current intensity, the temperature change of the four measured points are obvious, especially points (1) and (3). The magnitude of temperature increase is very large. Through the comparison of the above charts, firstly, it was described that in the current, the temperature of tested points rise faster, Secondly, In the four tested points, (1)、(2)、(3) are at high temperature, from the inner to the outer disk, the temperature distribution is a general trend of increased first and then decreased.

\section{Conclusion}

(1) Through temperature analysis which is based on the loading device, it can be concluded that in many conditions, real-time temperature data transmission part of the various parts of the dynamometer. Effect of temperature on magnetic rheological fluid dynamometer is obvious, but after grasp the regularity of the effect, the current can be adopted in practical application to fine tune the corresponding to the dynamometer attenuation compensation.

(2) Based on heat conduction theory, and analyzes the effect of temperature on properties of magneto-rheological transmission device by means of experimental method. The study found, when magneto-rheological transmission device temperature reaches the steady state, the minimum temperature point on the rotating shaft which is close to the transmission shaft, the highest point appears in the drive disc diameter between inside and outside, the temperature distribution is uniform; secondly, the yield stress of magnetic rheological fluid dynamic can reduce as the increase of the temperature, but because of the effect of reluctance of the magnetic circuit and extrusion enhancement ,the force transmission performance can slightly improve magneto-rheological transmission device.

\section{About the Authors}

Fang Yi was born in October.1989 in Hubei province. He got bachelor's degree of Mechanical Engineering in Yangtze University in 2012 and now is a Graduate student in Shanghai University of Engineering Science.

Luo Yiping was born in 1966. He gained a master's degree of Industrial Engineering in Shanghai Jiao Tong University in 1987. Now he is a professor in Shanghai University of Engineering Science.

Ren Hongjuan was born in 1978 in Shandong province. She gained a master's degree of Power Machinery and engineering in Shandong University in 2002. Now she is an associate professor in Shanghai University of Engineering Science.

\section{Acknowledgments}

This research was supported by the construction of professional platform for modern automobile service engineering in Shanghai city (XKCZ1214).

\section{References}

[1] Kordonski W I, "Magneto-rheological Effect as a Base of New Devices and Technologies," J Magnetism \&Magnetic Mat, vol.122, pp.395-398, 1993.

[2] Lan Wenkui, Zheng Ling, Li Yinong, et al, "FEM analysis of magnetic field of piston-type MR fluids damper," Transactions of the Chinese Society for Agricultural Machinery, vol. 38, no. 4, pp.142-145, 2007.

[3] Ma H R, Wen W J, Tam W Y, et al, "Dielectric electro rheological fluids: Theory and experiment," Adv Phys ,vol.52, pp.343 347, 2003.

[4] Winslow W M, "Method and Means for Translating Electrical Impulse into Mechanical Force," $U . S$. Patent No.2417850.1947.

[5] Rabinow J, "The Magnetic Fluild Cluth," AIEE Transactions, vol. 67 , pp.1308-1315, 1948.

[6] Park B J, Park CW, Yang SW, et al, "Core shell Typed Polymer Coated-carbonyl Iron Suspensions and Their Magneto-rheology," Journal of Physics : Conference Series, vol.149, no.1, pp.1-5, 2009.

[7] Barber D E, Carlson J D, "Performance Characteristics of Prototype MR Engine Mounts Containing LORD Glycol MR Fluids," Journal of Physics: Conference Series, vol.149, pp.1-4, 2009. 
[8] Chin B D, Park J H, Kwon M H, et a1, "Rheological Properties and Dispersion Stability of Magneto-rheological (MR) Suspensions," Rheol Acta, vol.40, no.3, pp.211-219, 2001 .

[9] Yang Shiqing, Zhang Wanli,"Rheological properties of magneto-rheological fluid," Functional material,vol.29, no.5, pp.550-552, 1998.

[10] Xie Fangwei, HouYoufu, "Transient thermal-stress coupling of friction pair of hydro-viscous drive device," Journal of Central South University: Science and Technology, vol.41, no.6, pp. 2201-2206, 2010.

[11] Li Zhongxian, Wu Linlin, XuLonghe, etc.. Structural design of MR damper and experimental study for performance of damping force $[\mathrm{J}]$. Earthquake engineering and engineering vibration, 2003,23(1):128-132.

[12] Wang Hongtao, Zhang Jinqiu, Bi Zhandong, etc.. Model and Analysis of Damping for Twin-tube Magneto-rheological Fluid Damper Based on Disc Type Orifice [J]. Journal of mechanical engineering, 2010,46(18):139-144.

[13] Wang Xiuyong, Sun Hongxin, Chen Zhengqing. Rotation shear magneto-rheological fluid damper design and mechanical model [J]. Journal of vibration and shock, 2010, 29 (10):77-81.

[14] $\mathrm{Lu} \mathrm{He,} \mathrm{Liu} \mathrm{Xinhua,} \mathrm{magneto-rheological} \mathrm{fluids} \mathrm{and} \mathrm{its}$ applications in mechanical engineering $[\mathrm{J}]$. Technology and application of manufacturing, 2013, (1):66-70.
[15] Kordonaky W.I., Garodkin S.R., Magneto-rheological Fluid Based Seal. In Bullogh W.A., Proc of the 5th Int. Conf. on ER Fluids, MR Suspensions and Associated Technology, Singapore: World Scientific, 1996, P.704-709.

[16] Tang $X$, Zhang $X$, Tao T. Flexible Fixture with Magneto-rheological Fluids, In: Tao R, Proc. Of the 7th Int. Conf. on ER Fluids, MR Suspensions and Associated Technology, Singapore: World Scientific, 2000, P.712-720.

[17] W.M I Kordonski, Adaptive Structures Base on Magneto-rheological Fluids, Proc. 3 Int. Conf. Adaptive Struct., ed. Wada, Natori and Breitbach. P.13-17, San Diego, CA, 1992.

[18] Jolly R, Bender W, Carlson J. Pro per ties and applications of commercial magneto-rheological fluids [J]. Journal of Intelligent Mateial Systems and Structures, 1999, 10: 5 13.

[19] Li H, Peng X, and Chen W. A Micro-to-macroscopic Analysis for the Yield Stress of Magneto-rheological Fluids, Proc. Int. Conf. Heterogeneoud Materials Mechanics, edited by Fan j, McDowell D and Huang K, Chongqing University Press, Chongqing, 2004: 276-280.

[20] Yi Zhou, Jianzuo, Ma, Dong Zuo. A Self-adaptive Fan Clutch for Automobile. New and Advanced Materials. Advanced Materials Research Vols. 197-198, 2011: 339-343 